\title{
Toothbrushing: to the best of one's abilities is possibly not good enough
}

\author{
Renate Deinzer ${ }^{1 *} \mathbb{D}$, Stefanie Ebel ${ }^{1}$, Helen Blättermann ${ }^{1}$, Ulrike Weik ${ }^{1}$ and Jutta Margraf-Stiksrud ${ }^{2}$
}

\begin{abstract}
Background: Weaknesses in toothbrushing performance can be seen when young adults are instructed to perform habitual toothbrushing. Nothing is known about toothbrushing behavior when instructed to perform to the best abilities. The present study analyzes such behavior and compares it to habitual behavior.

Methods: A random sample of $N=98$ young adults born in 1995 was examined in 2014/2015.They were asked to perform oral hygiene to the best of their abilities in front of a camera. Videos were analyzed regarding details of brushing behavior. A quality index was developed which describes the extent of the neglect of brushing on palatinal and vestibular surfaces. Data were compared to those of an earlier study of young adults (born in 1992, examined in $2011, N=101$ ) who were asked to perform oral hygiene as they habitually do.
\end{abstract}

Results: The 1995 cohort (best abilities) brushed their teeth significantly longer than the 1992 cohort (habitual brushing). This was due to significant longer brushing at vestibular and occlusal surfaces. Neglect of palatinal surfaces was similar in both cohorts. Groups did not differ regarding brushing movements. $40 \%$ of the brushing time on lateral surfaces was spent with scrubbing movements despite opposing advice in common oral hygiene instructions.

Conclusions: Toothbrushing to the best of one's abilities might still not be good enough. Young adults apparently lack a reasonable concept of what is meant by high quality toothbrushing. More efforts should thus be undertaken to explain them (and adults) this concept.

Keywords: Oral hygiene, Community dentistry, Dental education, Dental hygiene, Preventive dentistry, Behavioral science, Toothbrushing

\section{Background}

There is hardly any other health behavior in western communities that is performed by such a great portion of the population on a regular basis as oral hygiene behavior. This can be considered a major success of joint ventures by dental health professionals, health politicians and educational staff, who succeeded in establishing oral hygiene as a daily routine in the vast majority of the population $[1,2]$. Still, oral diseases, especially gingivitis and periodontitis, are very common in adults. Nearly everyone suffers from gingivitis and approximately $50 \%$ show at least some periodontal breakdown [2-6]. It thus appears that even though oral hygiene is performed on a regular basis, its quality tends to be low or at least

\footnotetext{
* Correspondence: renate.deinzer@mp.jug.de;

renate.deinzer@psycho.med.uni-giessen.de

'Department of Medicine, Justus-Liebig-University Giessen, Klinikstr. 29,

D-35392 Giessen, Germany

Full list of author information is available at the end of the article
}

insufficient with respect to oral health maintenance. A series of recent studies has shown that adults hardly ever manage to clean more than $30-40 \%$ of their gingival margins by means of tooth brushing and interproximal hygiene [7-12]. Thus, the question arises of why oral hygiene behavior is so inefficient.

In order to answer this question, a recent study analyzed the oral hygiene behavior of young adults who had just come of age [13]. On reaching this age, years of prophylactic programs aiming to bring them into a position of maintaining oral health during adulthood had concluded. These young adults were asked to demonstrate their habitual oral hygiene behavior while being filmed. Analyses showed that they brushed their teeth for more than $2 \mathrm{~min}$, but distributed brushing time unevenly among sextants and surfaces and neglected palatinal and lingual surfaces. Furthermore, even though the prophylactic programs teach children and adolescents to

(c) The Author(s). 2018 Open Access This article is distributed under the terms of the Creative Commons Attribution 4.0 International License (http://creativecommons.org/licenses/by/4.0/), which permits unrestricted use, distribution, and reproduction in any medium, provided you give appropriate credit to the original author(s) and the source, provide a link to the Creative Commons license, and indicate if changes were made. The Creative Commons Public Domain Dedication waiver (http://creativecommons.org/publicdomain/zero/1.0/) applies to the data made available in this article, unless otherwise stated. 
apply circular and/or vertical rather than horizontal brushing movements at lateral surfaces, horizontal movements were evident in most of them for a considerable period of time. Finally, only 50\% showed some interproximal hygiene behavior. In addition, this was considered inadequate in most cases [13].

While this study gave important insights into habitual oral hygiene behavior of young adults, nothing was learned about their behavior when they perform to the best of their abilities. From additional analyses it is already known that even then they do not manage to achieve oral cleanliness at most of their gingival margins [9]. However, nothing is known about what characterizes their oral hygiene behavior under such circumstances and whether and in what respect it differs from their habitual behavior.

The present study thus aimed to investigate young adults' oral hygiene behavior when they were asked to perform oral hygiene to the best of their abilities and to analyze behavioral differences from the former cohort of young adults [13] who had been asked to perform their habitual oral hygiene behavior.

\section{Methods}

The aim of the present study was to analyze oral hygiene behavior of young adults when they were asked to perform to the best of their abilities and to compare this behavior to behavior assessed in an earlier study [13] when participants were asked to perform as usual.

\section{Participants and general design}

Methods relevant for the comparison of the former cohort [13] and the current one were essentially the same and a detailed description will only be given for the current cohort. The study protocol of the former study has been described in detail elsewhere [13]. In order to enhance the comparability to the previous study [13], this study assessed oral hygiene behavior in young adults living in the same town (approx. 80,000 inhabitants) in central Germany. All inhabitants born in 1995 were invited. They had come of age a few months prior to the beginning of recruitment in August 2014. The assessments took place in dental examination rooms of the Institute of Medical Psychology, Justus-Liebig-University Giessen, Germany, from August 2014 to July 2015.

$N=98$ young adults ( 43 males and 55 females) participated in the present study. Details of recruitment are shown in the Additional file 1.

All participants fulfilled the inclusion criteria: 1) born in 1995; 2) being a resident of Giessen; 3) no training in any dental profession; 4) providing informed written consent, and also the exclusion criteria: 1) fixed orthodontic appliances; 2) cognitive or physical impairment that affects toothbrushing; 3) habitual use of a powered toothbrush; 4) removable dentures.

Participants were placed in front of a wash basin and a tablet computer. The tablet computer had an integrated front camera and it served both as a mirror for the participants and as a camera. All participants were provided with a standardized toothbrush and toothpaste. In contrast to the earlier study where participants were only offered $0.5 \mathrm{~m}$ of dental floss, the present participants were provided with a selection of different means for proximal hygiene (waxed and unwaxed dental floss, superfloss, interdental brushes). Participants were asked to clean their teeth to the best of their abilities and were left alone while performing oral hygiene. In the present study, some clinical data were also assessed prior to and immediately after oral hygiene. Most importantly, the marginal plaque index (MPI) [11] was assessed immediately after oral hygiene by a calibrated examiner (for details see Ebel et al. submitted). No corresponding data are available from the previous study [13]. Clinical data are discussed in detail elsewhere [14]. Furthermore, some questionnaire data were assessed regarding psychological parameters which will not be discussed in the current analyses. The highest degree of education of the participants' parents was assessed as a measure of socioeconomic status. Two categories were formed for analyses: university entrance diploma or not.

\section{Observed oral hygiene behavior}

The videos were analyzed by two independent calibrated examiners (HB und SE) using the software Mangold Interact 14 (Mangold International $\mathrm{GmbH}$, Arnstorf, Germany). Brushing hand was coded and the examiners watched the video multiple times (also in slow motion) in order to code further behavioral categories. Calibration was provided by five videos of individuals not involved in the present study.

In adulthood, caries manifests primarily at lateral and proximal surfaces [15-17]. The risk of developing gum disease and periodontitis increases and exceeds that of caries $[2,5]$. Therefore, special emphasis was given to brushing behavior at lateral surfaces. Analyses regarding brushing movements and precise localization of brushing (sextants) were confined to them. To ensure that these analyses were not contaminated by occlusal brushing, lateral brushing was only assumed if both raters agreed that it was not occlusal. Thus, both examiners coded tooth contact time (time while toothbrush touches the teeth, without rinsing, spitting, tongue cleaning or breaks) and surfaces (vestibular, palatinal and occlusal) for all participants. SE carried out all further ratings and confined these to the palatinal and vestibular surfaces. These were: brushing movements (horizontal i.e. scrubbing, vertical, circular, modified Bass 
technique) and sextants (sextant 1 to 6 , and, at vestibular surfaces, also concurrent brushing of antagonistic sextants, i.e. 1 and 6, 2 and 5, 3 and 4, respectively). Concurrent brushing of antagonistic sextants was coded when participants closed the mandibles while brushing. For further analyses, the brushing time of two sextants brushed concurrently was distributed in equal parts to both sextants.

To assess whether codings of sextants and movements remained reliable over time, double codings of a random sample of films were performed by HB. SE did not know which films were double coded and HB did not know SE's codings. Intraclass correlations of double codings of the various observational categories were all above 0.801 .

In the former study [13], all behavioral parameters were assessed by two independent examiners. Their intraclass correlations regarding the different parameters exceeded ICC $=0.865$. For the present analyses, we computed the mean values of these double codings, since aggregating double codings further increases their reliability.

It was also intended to assess interdental cleaning (i.e. any application of devices for interdental cleaning in interdental spaces). However, only 15 participants performed interdental cleaning. Furthermore, most of these applied them only in some interdental spaces. We therefore refrained from any further analysis of this behavior.

A main result of the former study was that participants neglected surfaces and sextants while brushing. In the present analysis, a scoring system was thus developed allowing further description and analysis of the quality of toothbrushing at palatinal and vestibular surfaces (Quality index of toothbrushing regarding brushing time in sextants: QIT-S; for details see Table 1).

\section{Statistics}

The intended significance level was $\alpha=5 \%$. For parametric data, comparisons within a cohort were performed by paired $\mathrm{t}$-tests and group comparisons by t-tests for independent measures. The latter are reported together with Cohen's d. If Levene's test for comparison of variances reached the significance level, the $t$-statistic for unequal variances was chosen. For non-parametric data, results of exact rank tests or exact $\mathrm{Chi}^{2}$ tests are reported. All analyses were run with IBM SPSS Statistics Version 24.

\section{Results}

For ease of reading, the current cohort will be the 1995 cohort and the former cohort [13] will be referred to as the 1992 cohort. Two participants of the 1995 cohort had to be excluded from further analyses: one due to restricted visibility during brushing, another because of
Table 1 The Quality index of toothbrushing regarding brushing time in sextants (QIT-S $)$

\begin{tabular}{ll}
\hline QIT-S-0 & 0 sextants brushed by more than $1 \mathrm{~s}$ \\
QIT-S-1 & 1 sextants brushed by more than $1 \mathrm{~s}$ \\
QIT-S-2 & 2 sextants brushed by more than $1 \mathrm{~s}$ \\
QIT-S-3 & 3 sextants brushed by more than $1 \mathrm{~s}$ \\
QIT-S-4 & 4 sextants brushed by more than $1 \mathrm{~s}$ \\
QIT-S-5 & 5 sextants brushed by more than $1 \mathrm{~s}$ \\
QIT-S-6 & all sextants brushed by more than $1 \mathrm{~s}$ \\
QIT-S-7 & all sextants brushed by more than $3.5 \mathrm{~s}$ \\
QIT-S-8 & all sextants brushed by more than $5 \mathrm{~s}$ \\
QIT-S-9 & all sextants brushed by more than 7.5
\end{tabular}

${ }^{a}$ The QIT-S index represents a rank-scaled measure allowing for the differential analysis of brushing time distribution at palatinal and vestibular sites, respectively. Brushing time of less than $1 \mathrm{~s}$ within a sextant is considered neglect of this sextant. QIT-S-0 - QIT-S-6 describe the expansion of this neglect. The highest score (QIT-S-9) is deduced from a recommended total brushing time of 2 min (e.g. 19 [19]; 18 [18]; 20 [20]) and an estimation of $30 \mathrm{~s}$ brushing time for occlusal surfaces as occlusal brushing is easier and can be done with greater movements than palatinal or vestibular brushing. Thereby, $45 \mathrm{~s}$ remain for the palatinal and vestibular surfaces, respectively. An even distribution of this time across sextants results in $7.5 \mathrm{~s}$ per sextant. As 3.5 represents roughly half of 7.5 this is taken as further step (QIT-S-7) and as $5 \mathrm{~s}$ per sextant might already be considered fair this was taken as another step (QIT-S-8)

her extremely extended brushing time (573.4 s, 4 standard deviations above the mean) which also led to distortions of the reliability estimates of observational measures.

In the 1995 cohort, percentage of marginal sections showing persistent plaque (MPI; 11) immediately after oral hygiene was $69.4 \% \pm 12.3$ regarding the whole mouth, and $60.9 \% \pm 15.3$ and $78.0 \% \pm 12.2$ regarding vestibular and palatinal sites, respectively. No such data is available for the 1992 cohort.

Table 2 shows the characteristics of the two cohorts with respect to sex, parents' education and brushing hand. No significant differences between the cohorts were found.

Table 3 shows the brushing behavior of the two cohorts. Both cohorts spent most of the brushing time on vestibular and occlusal surfaces. Significantly less brushing time was spent on palatinal than on vestibular surfaces in both cohorts (all $p<.001$ ). Groups did not differ with respect to brushing movements on lateral surfaces.

Table 2 Characteristics of the two cohorts

\begin{tabular}{llll}
\hline & 1995 cohort & 1992 cohort & $\mathrm{p}$ (exact test) \\
\hline sex & male/female & male/female & 0.491 \\
& $42 / 54$ & $43 / 58$ & \\
at least one parent has & no UED/UED & no UED/UED & 0.085 \\
$\begin{array}{l}\text { university entrance } \\
\text { diploma (UED) }\end{array}$ & $36 / 60$ & $51 / 50$ & \\
brushing hand in video & right/left/both & right/left/both & 0.886 \\
& $85 / 7 / 4$ & $85 / 10 / 6$ & \\
\hline
\end{tabular}


Table 3 Brushing behavior within the two cohorts

\begin{tabular}{|c|c|c|c|c|c|}
\hline & $\begin{array}{l}1995 \text { cohort }(N=96) \\
\text { Mean }(S D)\end{array}$ & $\begin{array}{l}1992 \text { cohort } \\
(N=101) \\
\text { Mean (SD) }\end{array}$ & $t(195)$ & $d$ & $p$ \\
\hline \multicolumn{6}{|l|}{ Tooth contact time (s) } \\
\hline overall & $206.7(84.0)$ & $155.3(70.8)$ & 4.66 & .664 & $<.001$ \\
\hline vestibular & $91.3(40.7)$ & $70.9(31.2)$ & 3.94 & .565 & $<.001$ \\
\hline palatinal & $30.6(30.9)$ & $26.0(27.9)$ & 1.10 & .157 & .272 \\
\hline occlusal & $84.8(46.0)$ & $58.4(34.2)$ & 4.55 & .654 & $<.001$ \\
\hline \multicolumn{6}{|l|}{ Percentage of time lateral surfaces are brushed by } \\
\hline horizontal (scrubbing) brushing movements & $39.7(28.8)$ & $39.7(30.3)$ & .002 & $<.001$ & .998 \\
\hline circular brushing movements & $47.0(27.3)$ & $44.8(29.5)$ & .548 & .078 & .584 \\
\hline
\end{tabular}

Circular and horizontal brushing predominated, while vertical brushing was rarely shown for longer periods of time. Only 1 person of the 1995 cohort applied the modified Bass technique.

Figure 1 shows the QIT-S scores. QIT-S palatinal was significantly lower than QIT-S vestibular in both groups (exact $p<.001)$. Groups did not differ with respect to QIT-S palatinal (exact $p=.331$ ) while the 1992 cohort showed lower QIT-S vestibular scores than the 1995 cohort (exact $p=.002$ ).

A further analysis was run in order to better understand the differences between the cohorts regarding vestibular brushing: The percentage by which these groups distributed their vestibular brushing time to right (sextants 1 and 6), left (sextants 2 and 5) and anterior (sextants 3 and 4) surfaces was compared. Cohorts did not differ with respect to brushing time at left surfaces, but the 1995 cohort showed prolonged brushing of anterior and right surfaces (see Table 4).

\section{Discussion}

One important aim of oral health education of children and adolescents is to enable them to employ proper oral hygiene until they come of age. However, recent studies have shown that the plaque removal capability of young adults still tends to be low [7-10]. A major aim of the present study was thus to explore their oral hygiene skills. Therefore, participants were observed while performing oral hygiene to the best of their abilities. In order to differentiate their behavior with respect to distribution of brushing time, a quality index (QIT-S) was developed.

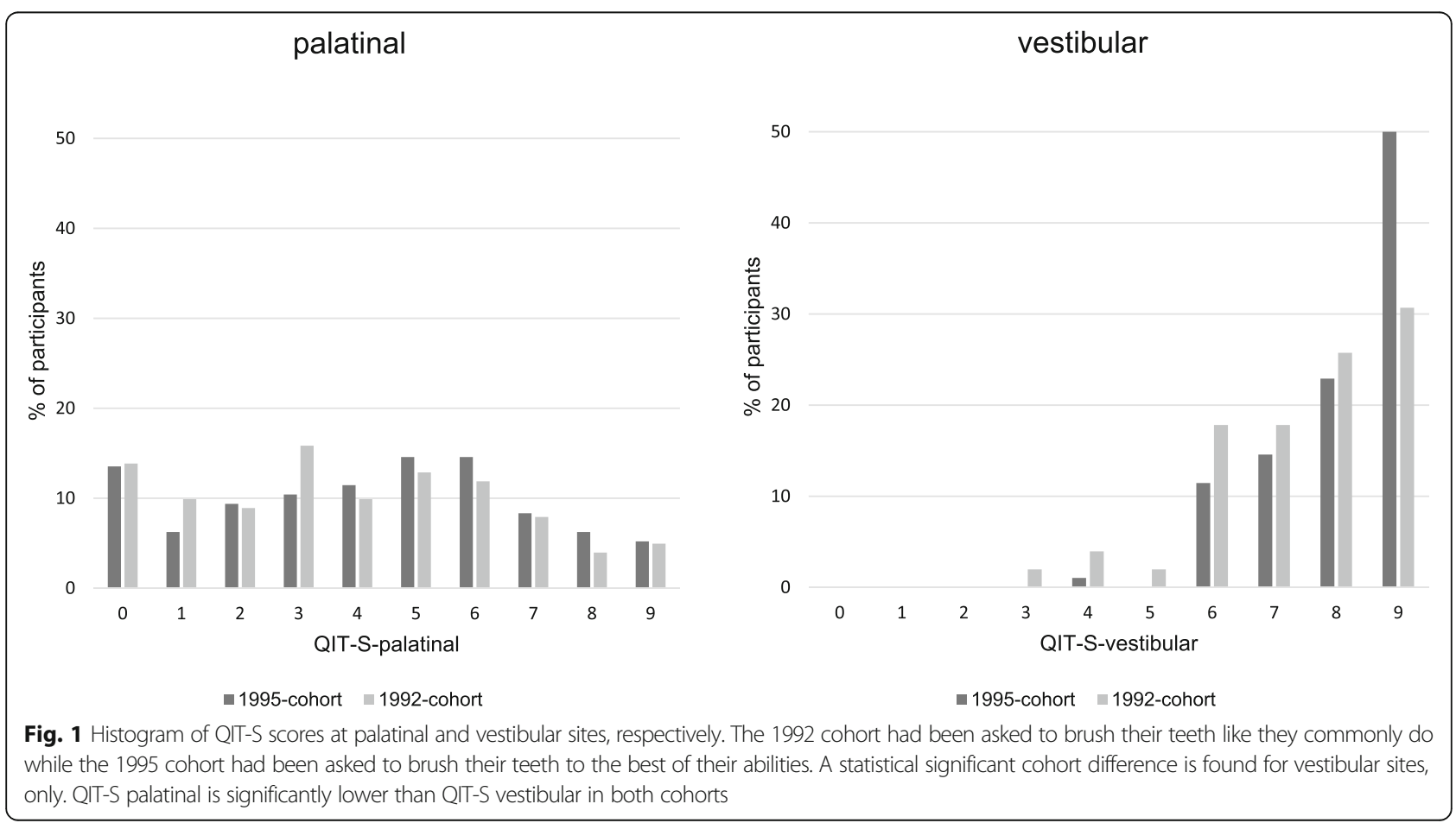


Table 4 Brushing time across vestibular surfaces

\begin{tabular}{llllll}
\hline \multicolumn{1}{c}{$\begin{array}{l}1995 \text { cohort }(N=96) \\
\text { Mean (SD) }\end{array}$} & $\begin{array}{l}1992 \text { cohort } \\
(N=101) \\
\text { Mean (SD) }\end{array}$ & t (195) & d & $p$ \\
\hline $\begin{array}{l}\text { Brushing time (seconds) } \\
\text { anterior }\end{array}$ & $39.3(21.3)$ & $24.7(15.6)$ & 5.5 & .788 & $<.001$ \\
right & $26.6(15.4)$ & $19.7(10.2)$ & 3.7 & .532 & $<.001$ \\
left & $25.2(13.9)$ & $26.5(13.5)$ & -0.6 & -.088 & .536 \\
\hline
\end{tabular}

The good news from these observations is that young adults, when asked to perform oral hygiene to the best of their abilities, spent an average of 3:20 min brushing. This is more than $60 \%$ above common recommendations (see for example [18-20]) and suggests that they were motivated to give their best.

The bad news is that they distributed this brushing time neither evenly nor efficiently: These young adults brushed occlusal surfaces nearly 3 times longer than palatinal surfaces, even though gum disease and even caries in adults [15-17] originate at lateral surfaces. Furthermore, $80 \%$ of the study sample skipped at least one sextant when brushing palatinal surfaces (QIT-S palatinal $\leq 5$ ); only $5 \%$ brushed all palatinal sextants for more than $7.5 \mathrm{~s}$ (QIT-S palatinal =9). Vestibular sextants, on the other hand, were hardly ever skipped (only by one person), and were brushed for more than $7.5 \mathrm{~s}$ (QIT-S vestibular $=9$ ) by $50 \%$ of the study sample.

Regarding brushing techniques, oral hygiene education usually teaches children to brush lateral surfaces either by circular or by vertical but not by scrubbing movements (see for example [21-23]). Still, the present sample spent nearly $40 \%$ of the brushing time on lateral surfaces scrubbing.

Considering that the participants of this study performed oral hygiene to the best of their abilities, the question arises as to what characterizes this behavior "to the best of one's abilities" as compared to one's common behavior. A second aim of the present study was thus to compare its results to a former study which had analyzed young adults' common oral hygiene behavior [13]. This comparison elicited three important results: First, even though the total brushing time of the "to the best of one's abilities" group exceeded that of the "common oral hygiene" group by more than a minute, the time spent brushing palatinal surfaces remained the same. Secondly, maximizing one's efforts apparently did not result in altering one's brushing technique. The cohorts did not differ with respect to the applied brushing movements. The only improvement of quality in oral hygiene behavior was seen with respect to vestibular surfaces: Neglect of surfaces (QIT-S vestibular $\leq 5$ ) decreased, whereas the portion of participants achieving the highest quality score (QIT-S vestibular =9) increased (see Fig. 1).
A closer inspection of the distribution of brushing time across vestibular surfaces indicated that maximizing one's efforts resulted in a disproportionately high increase in brushing time of anterior surfaces: Interestingly, these surfaces were already brushed for the longest time when people were asked to show their "common" hygiene behavior.

Summarizing, these results indicate that young adults, when asked to brush their teeth to the best of their abilities, tend to increase efforts within regions they already brush for a disproportionately long time (i.e. occlusal sites, vestibular sites, and within vestibular sites, anterior teeth), but continue to neglect palatinal sites. Furthermore, a considerable portion of brushing time remains spent scrubbing, irrespective of opposing content of oral hygiene teaching.

Considering these results, one has to doubt that young adults have adopted a reasonable concept of what is meant by high quality oral hygiene behavior. Their concept appears to be confined to brushing time. Neither did they seem to be aware of the meaning of brushing systematics (in order not to forget any surfaces), nor did they alter brushing techniques. The first point is especially striking, since neglecting whole regions while brushing inevitably results in poor plaque removal. Regarding the second point, one should keep in mind that strong scientific evidence demonstrating the superiority of one brushing technique above another is lacking $[7,24]$. Still, dental advice commonly discourages people from scrubbing. Thus, one would have expected that the percentage of time spent scrubbing decreases when people try to perform high quality brushing.

Some limitations of the present study should be considered. First of all, self-selection of the participants may have biased results. This, however, presumably resulted in an overestimation of the toothbrushing quality of the cohort, as one would expect mainly those who doubt their competence to reject participation in an oral hygiene study. Secondly, it remains unclear as to what degree study results can be generalized to other regions of the world. Instead, it would be worth exploring whether similar or differing results would be observed in other nations with differing oral hygiene education programs. The present research demonstrates how important it is to analyze oral hygiene behavior more closely in order to understand hygiene deficits. Thirdly, the comparison between the two cohorts is merely quasi-experimental, thus not allowing for firm causal conclusions. While groups are perfectly comparable regarding age at the time of examination and with respect to demographic characteristics, concerns might arise regarding the following factors: year in which the participant was born and the examination took place, oral hygiene devices, different examiners. However, there is only a three-year 
gap between the two studies, the hygiene devices were very similar, and all examiners were calibrated by the same method and very good intraclass correlations were achieved. Thus, the comparability of the cohorts appears to be good enough to justify at least some reasoning about the meaning of the instruction (to the best of one's abilities vs. common behavior) for oral hygiene behavior. Finally, one might question whether the behavioral deficits observed here reflect deficits in oral hygiene motivation rather than in oral hygiene skills. However, participants performed oral hygiene in a dental setting, they were asked to perform to the best of their abilities, they knew that they would be given a clinical examination afterwards and they brushed their teeth for far longer than usually recommended. This all argues against the assumption that the study results reflect motivational deficits rather than skill deficits.

Still, future research is needed to overcome the limitations of this analysis. Most importantly, the effect of different oral hygiene instructions should be assessed within a randomized controlled trial (RCT) and plaque after oral hygiene should be assessed in that study and related to hygiene behavior. Future studies should also focus on other age groups.

\section{Conclusion}

Concluding, the present study demonstrates that - at least in young German adults - the demand to improve one's oral hygiene might be useless as long as it is not explained in detail what exactly has to be improved. The observed distribution of brushing time across regions indicates that young adults have a poor concept of what is important while brushing. They appear to prefer those sites which are visible (vestibular, mainly anterior). They also prefer occlusal surfaces and thereby appear to preserve a principle which had some justification in childhood but loses its validity in coming of age: Children learn that caries is the most important oral disease and that it manifests primarily at occlusal surfaces. This is true for children. In adulthood, however, caries manifests primarily at lateral and proximal surfaces [15-17] and the risk of developing gum disease and periodontitis increases and exceeds that of caries [2,5]. It is important, that this change of principles is understood when entering adulthood. The current study indicates, however, that this is not the case. This assumption is supported by another representative study [25]. In that study, even middle-aged adults believed caries to be more important and prevalent than periodontal disease. Further, they indicated that it would be very important to clean one's occlusal surfaces in order to prevent periodontitis [25]. More efforts should thus be undertaken to explain to adolescents (and adults) this change of principle.
The present investigation shows that many future efforts are necessary in order to better understand peoples' oral hygiene behavior and how they could be taught the capability of achieving oral cleanliness. This is not impossible as demonstrated by a recent study showing that dental professionals already manage to keep their teeth clean and healthy [26]: Thus, it can be learned.

\section{Additional file}

\begin{abstract}
Additional file 1: Appendix: Flow diagram of participant recruitment. Delineates the recruitment of the two cohorts in detail: number of persons contacted, responding/not responding, excluded, included, examined, with no appointment. (DOCX $182 \mathrm{~kb}$ )
\end{abstract}

\section{Abbreviations}

HB: Helen Blättermann (co-author); ICC: Intraclass correlation; MPI: Marginal Plaque Index; QIT-S: Quality Index of toothbrushing regarding brushing time in sextants; RCT: Randomized controlled trial; SD: Standard deviation; SE: Stefanie Ebel (co-author)

\section{Acknowledgements}

This research was financed by the institutional budget of RD. We greatly appreciate the valuable assistance of Dr. Daniela Harnacke in planning and conducting this research. All authors report no perceived or actual conflicts of interest.

Funding

The study was financed by the institutional budget of the corresponding author.

\section{Availability of data and materials}

The datasets used and/or analysed during the current study are available from the corresponding author on reasonable request. For privacy reasons, however, individual data allowing for the identification of participants (e.g. videos) cannot be made available.

\section{Guidelines}

This study conforms to STROBE Guidelines.

\section{Authors' contributions}

$\mathrm{RD}$ and JMS developed the concept and design of this study. HB contributed to acquisition of clinical and behavioral data, SE and HB contributed to analysis of behavioral data, RD, UW, and SE performed statistical analyses, all authors contributed to the interpretation of the data. RD and JMS drafted the manuscript. All authors critically revised the manuscript and gave final approval. RD and SE revised the manuscript in response to reviewers'and editors' comments.

Ethics approval and consent to participate

The study protocol for the current study was conducted according to the principles of the Declaration of Helsinki and was approved by the local Ethics Committee of the Medical Faculty of the University of Giessen (AZ 79/14). All participants provided informed written consent

Consent for publication

Not applicable

Competing interests

All authors declare that they have no competing interests.

\section{Publisher's Note}

Springer Nature remains neutral with regard to jurisdictional claims in published maps and institutional affiliations. 


\section{Author details}

'Department of Medicine, Justus-Liebig-University Giessen, Klinikstr. 29, D-35392 Giessen, Germany. ${ }^{2}$ Department of Psychology, Philipps University of Marburg, Gutenbergstr. 18, D-35032 Marburg, Germany.

Received: 2 July 2018 Accepted: 5 October 2018

Published online: 19 October 2018

\section{References}

1. Honkala S, Vereecken C, Niclasen B, Honkala E. Trends in toothbrushing in 20 countries/regions from 1994 to 2010. Eur J Pub Health. 2015;25(Suppl 2):20-3.

2. Jordan AR, Micheelis W. Fünfte Deutsche Mundgesundheitsstudie (DMS V) [Fifth German Oral Health Study]. Köln, Germany: Deutscher Zahnärzte Verlag DÄV; 2016. (Materialienreihe / Institut der Deutschen Zahnärzte; vol 35)

3. Hoffmann T. Parodontalerkrankungen [Periodontal Diseases]. In: Micheelis W, Schiffner U, editors. Vierte Deutsche Mundgesundheitsstudie (DMS IV) [Fourth Oral Healh Study (DMS IV)]. Köln, Germany: Deutscher Zahnärzte Verlag DÄV; 2006. p. 266-89. (Materialienreihe / Institut der Deutschen Zahnärzte; vol. 31).

4. Petersen $\mathrm{PE}$, Ogawa $\mathrm{H}$. The global burden of periodontal disease: towards integration with chronic disease prevention and control. Periodontol. 2012; 60(1):15-39.

5. Jin $\sqcup$, Lamster IB, Greenspan JS, Pitts NB, Scully C, Warnakulasuriya S. Global burden of oral diseases: emerging concepts, management and interplay with systemic health. Oral Dis. 2016;22(7):609-19.

6. Tonetti MS, Jepsen S, Jin L, Otomo-Corgel J. Impact of the global burden of periodontal diseases on health, nutrition and wellbeing of mankind: a call for global action. J Clin Periodontol. 2017:44(5):456-62.

7. Harnacke D, Beldoch M, Bohn G-H, Seghaoui O, Hegel N, Deinzer R. Oral and written instruction of oral hygiene: a randomized trial. J Periodontol. 2012a;83(10):1206-12.

8. Harnacke D, Mitter S, Lehner M, Munzert J, Deinzer R. Improving oral hygiene skills by computer-based training: a randomized controlled comparison of the modified bass and the Fones techniques. PLoS One. 2012b;7(5):e37072

9. Harnacke D, Winterfeld T, Erhardt J, Schlueter N, Ganss C, Margraf-Stiksrud J, et al. What is the best predictor for oral cleanliness after brushing? Results from an observational cohort study. J Periodontol. 2015;86(1):101-7.

10. Harnacke D, Stein K, Stein P, Margraf-Stiksrud J, Deinzer R. Training in different brushing techniques in relation to efficacy of oral hygiene in young adults - a randomised controlled trial. J Clin Periodontol. 2016:43(1):46-52.

11. Deinzer R, Jahns $S$, Harnacke D. Establishment of a new marginal plaque index with high sensitivity for changes in oral hygiene. J Periodontol. 2014; 85(12):1730-8

12. Deinzer R, Harnacke D, Mengel R, Telzer M, Lotzmann U, Wöstmann B. Effectiveness of computer based training (CBT) on toothbrush skills of patients treated with crowns. A randomized control trial. J Periodontol. 2016:87(11):1333-42.

13. Winterfeld T, Schlueter N, Harnacke D, Illig J, Margraf-Stiksrud J, Deinzer R, et al. Toothbrushing and flossing behaviour in young adults-a video observation. Clin Oral Investig. 2015;19(4):851-8.

14. Ebel S, Blättermann H, Weik U, Margraf-Stiksrud J, Deinzer R. High plaque levels after thorough toothbrushing - what impedes efficacy? submitted.

15. Menghini GD, Steiner M, Marthaler TM, Weber RM. Rückgang der Kariesprävalenz bei Schweizer Rekruten von 1970 bis 1996 [decrease in the prevalence of caries in Swiss recruits from 1970 to 1996]. Schweiz Monatsschr Zahnmed. 2001;111: 410-6.

16. Stenlund H, Mejàre I, Källestål C. Caries incidence rates in Swedish adolescents and young adults with particular reference to adjacent approximal tooth surfaces: a methodological study. Community Dent Oral Epidemiol. 2003;31(5):361-7.

17. Mejàre I, Stenlund H, Zelezny-Holmlund C. Caries incidence and lesion progression from adolescence to young adulthood: a prospective 15-year cohort study in Sweden. Caries Res. 2004;38(2):130-41.

18. American Dental Association. General Recommendations for the Prevention of Caries and Gingivitis; 2018 [cited 2018 Jan 31]. Available from: URL: https://www.ada.org/en/member-center/oral-health-topics/home-care.

19. European Federation of Periodontology. Guidelines for effective prevention of periodontal diseases; 2017 [cited 2018 Jan 31]. Available from: URL: https://www.efp.org/perioworkshop/workshop-2014/quidelines/Preventionof-periodontal-diseases-guidance-for-dental-surgeons.pdf.
20. World Dental Federation. Caries Prevention and Management Chairside Guide; 2018 [cited 2018 Jan 31]. Available from: URL: https://www.fdiworlddental. org/sites/default/files/media/resources/2017-fdi_cpp-chairside_guide.pdf.

21. Canadian Dental Association. Flossing \& Brushing; 2018 [cited 2018 Feb 12]. Available from: URL: http://www.cda-adc.ca/en/oral_health/cfyt/dental_care/ flossing_brushing.asp.

22. Deutsche Arbeitsgemeinschaft für Jugendzahnpflege [German working group for adolescent dental care]. Wie soll mein Kind sich die Zähne putzen. [How should my child brush his/her teeth.]; 2013 [cited 2018 Feb 12]. Available from: URL: http://www.daj.de/FAQ.17.0.html.

23. Deutsche Gesellschaft für Zahn. Mund und Kieferheilkunde [German Society for Dental and Oral Medicine]: Häusliche Prophylaxe [Home dental prophylaxis]; 2012. cited 2018 Feb 12]. Available from: URL: https://www.zahnmedizinischepatienteninformationen.de/documents/10157/1129556/268572_1567347_H\% C3\%A4usliche+Prophylaxe.pdf

24. Muller-Bolla M, Courson F, Manière-Ezvan A, Viargues P. Toothbrushing: which methods to use? Rev Odont Stomat. 2011;40:239-60.

25. Deinzer R, Micheelis W, Granrath N, Hoffmann T. More to learn about: periodontitis-related knowledge and its relationship with periodontal health behaviour. J Clin Periodontol. 2009:36(9):756-64.

26. Deinzer R, Schmidt R, Harnacke D, Meyle J, Ziebolz D, Hoffmann T, et al. Finding an upper limit of what might be achievable by patients: oral cleanliness in dental professionals after self-performed manual oral hygiene. Clin Oral Invest. 2018;22(2):839-46.

\section{Ready to submit your research? Choose BMC and benefit from:}

- fast, convenient online submission

- thorough peer review by experienced researchers in your field

- rapid publication on acceptance

- support for research data, including large and complex data types

- gold Open Access which fosters wider collaboration and increased citations

- maximum visibility for your research: over $100 \mathrm{M}$ website views per year

At BMC, research is always in progress.

Learn more biomedcentral.com/submissions 